\title{
Comparison of ECG-based physiological markers for hypoxia in a preterm ovine model
}

\author{
Alex Zwanenburg ${ }^{1,2,3}$, Ben JM Hermans s, ${ }^{1,4}$, Peter Andriessen ${ }^{5,6}$, Hendrik J Niemarkt ${ }^{5}$, Reint K Jellema ${ }^{5}$, \\ Daan RMG Ophelders ${ }^{2,5}$, Rik Vullings ${ }^{8}$, Tim GAM Wolfs ${ }^{2,5,7}$, Boris W Kramer ${ }^{2,5,7}$ and Tammo Delhaas ${ }^{1,3}$
}

BACKGROUND: Current methods for assessing perinatal hypoxic conditions did not improve infant outcomes. Various waveform-based and interval-based ECG markers have been suggested, but not directly compared. We compare performance of ECG markers in a standardized ovine model for fetal hypoxia.

METHODS: Sixty-nine fetal sheep of 0.7 gestation had ECG recorded $4 \mathrm{~h}$ before, during, and $4 \mathrm{~h}$ after a 25 -min period of umbilical cord occlusion (UCO), leading to severe hypoxia. Various ECG markers were calculated, among which were heart rate (HR), HR-corrected ventricular depolarization/ repolarization interval (QT ), and ST-segment analysis (STAN) episodic and baseline rise markers, analogue to clinical STAN device alarms. Performance of interval- and waveform-based ECG markers was assessed by correlating predicted and actual hypoxic/normoxic state.

RESULTS: Of the markers studied, HR and QT demonstrated high sensitivity ( $\geq 86 \%)$, specificity $(\geq 96 \%)$, and positive predictive value (PPV) $(\geq 86 \%)$ and detected hypoxia in $\geq 90 \%$ of fetuses at 4 min after UCO. In contrast, STAN episodic and baseline rise markers displayed low sensitivity $(\leq 20 \%)$ and could not detect severe fetal hypoxia in 65 and $28 \%$ of the animals, respectively.

CONCLUSION: Interval-based HR and QT markers could assess the presence of severe hypoxia. Waveform-based STAN episodic and baseline rise markers were ineffective as markers for hypoxia.

C ontinuous monitoring of the fetal heart rate (HR) is assumed to provide clinical information about the wellbeing of the fetus (1). Cardiotocography (CTG) allows noninvasive real-time assessment of fetal HR and its response to uterine contractions and is nowadays used in clinics worldwide. However, HR patterns in CTG are often not predictive for hypoxia (2). Introduction of CTG in clinics did not significantly reduce occurrences of neonatal death or cerebral palsy but did lead to an increase in caesarean sections (1). Consequently, analysis of the ECG ST waveform (STAN) was proposed as an additional analysis method. STAN assesses hypoxia-induced changes in the ST waveform of the ECG (3) and operates in conjunction with CTG. Implementation of STAN in clinical practice was initially associated with a beneficial reduction in hypoxia-induced outcomes (metabolic acidosis and moderate/ severe neonatal encephalopathy) compared to CTG alone (4). However, recent meta-analyses of clinical trials demonstrated no clear advantage of STAN with CTG over CTG alone with respect to primary outcomes $(5,6)$.

STAN markers are based on the shape of the ECG waveform. Other ECG markers for hypoxia detection have been suggested, namely, atrioventricular (AV) conduction delay (PR) $(7,8)$, the relationship of AV delay and HR (PR/RR and PR-HR) $(9,10)$ and ventricular activation and repolarization (QT) (11) and HR-corrected QT ( $\mathrm{QT}_{c, \text { Bazett }}$ and $\left.\mathrm{Qt}_{c, \text { Hodges }}\right)$, as well as heart rate variability (HRV) in the temporal and frequency domain. These markers are all interval-based, i.e., they use time intervals between characteristic points in the ECG. A major difference between waveform-based markers and interval-based markers is the dependency of the former on the projection angle of the ECG lead with the heart vector. Variation in orientation of the heart vector can lead to different results for waveform-based markers. An example based on the relative height of the T-wave with respect to the QRS complex (T/QRS ratio) is demonstrated in Figure 1. Interval-based markers are less dependent on differences in projection angle and might perform better in detecting fetal hypoxia.

Direct comparison of ECG markers is hampered because the different markers have previously been studied under different circumstances. Therefore, we compare performance of waveform- and interval-based ECG markers for hypoxia in a standardized preclinical ovine fetal model of hypoxia-ischemia.

'Department of Biomedical Engineering, Maastricht University, Maastricht, The Netherlands; ${ }^{2}$ MHeNs School for Mental Health and Neuroscience, Maastricht University, Maastricht, The Netherlands; ${ }^{3}$ CARIM School for Cardiovascular Diseases, Maastricht University, Maastricht, The Netherlands; ${ }^{4}$ Department of Technical Medicine, University of Twente, Enschede, The Netherlands; ${ }^{5}$ Department of Pediatrics, Maastricht University Medical Centre, Maastricht, The Netherlands; ${ }^{6}$ Department of Pediatrics, Máxima Medical Centre, Veldhoven, The Netherlands; ${ }^{7}$ GROW School for Oncology and Developmental Biology, Maastricht University, Maastricht, The Netherlands; ${ }^{8}$ Signal Processing Systems Group, Department of Electrical Engineering, Eindhoven University of Technology, Eindhoven, The Netherlands. Correspondence: Tammo Delhaas (tammo.delhaas@maastrichtuniversity.nl)

Received 30 July 2015; accepted 24 November 2015; advance online publication 23 March 2016. doi:10.1038/pr.2016.21 


\section{Articles | Zwanenburget al.}

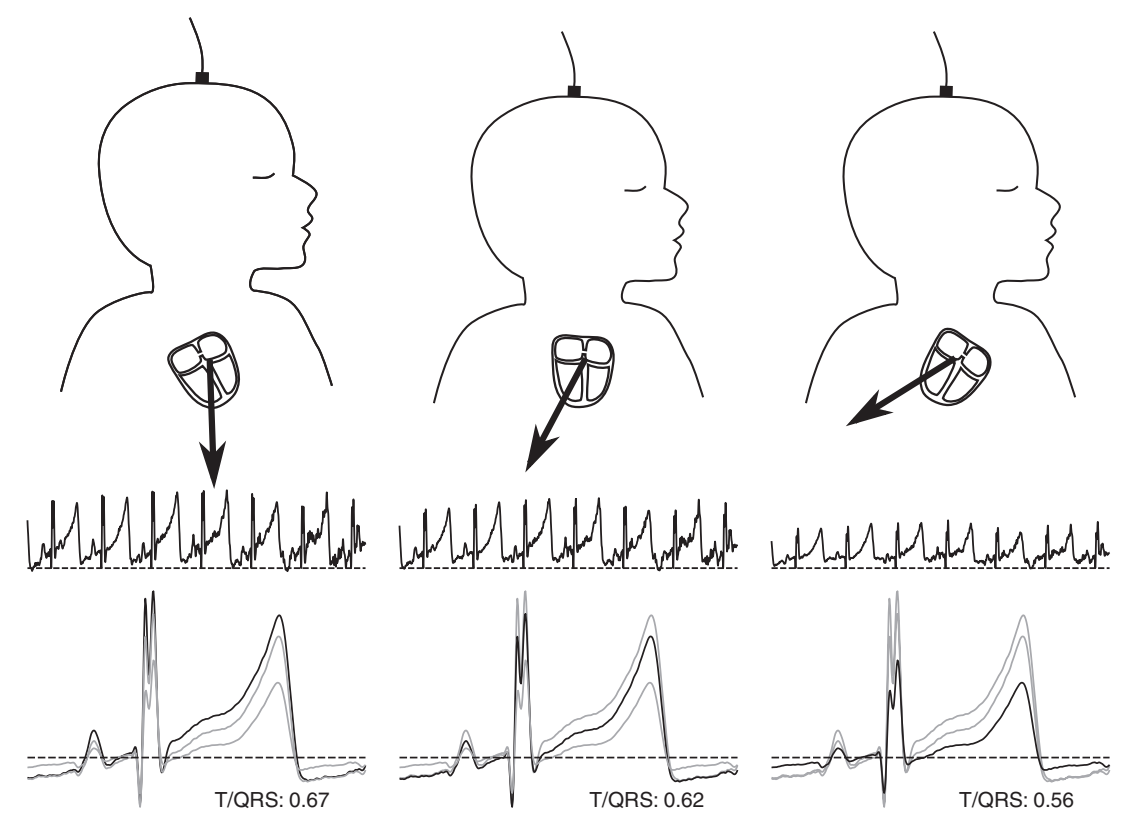

Figure 1. Influence of heart axis orientation on waveform-based T/QRS values. The same signal is projected to simulate a rotation of the heart axis by 30 and 60 degrees to the right. This affects the shape of the ECG waveform and the T/QRS ratio, defined as the maximum deviation of the T wave with regard to baseline divided by the amplitude of the QRS complex.

\section{RESULTS}

Presence of Umbilical Cord Occlusion Induced Hypoxia-Ischemia Blood gas measurements confirm a clinically relevant umbilical cord occlusion (UCO), with a decrease in partial oxygen pressure, an increase in partial carbon dioxide pressure, $\mathrm{pH}<7.1$ (Supplementary Figure $\mathbf{S 1}$ online), and bradycardia (Supplementary Figure S2 online). HR accelerates to above normal values within the first $15 \mathrm{~min}$ after resolution of the UCO. It then returns to almost normal values within the first hour after UCO and subsequently gradually increases again. Arterial $\mathrm{pH}$ values recover within the first hour. Arterial blood oxygen content directly rises after UCO and remains elevated during the first hour after UCO, whereas carbon dioxide content decreases to normal values within $10 \mathrm{~min}$.

\section{Classification Performance of ECG Markers}

Interval-based markers for hypoxia generally performed better than waveform-based markers. HR, PR/RR ratio, and HR-corrected QT ${ }_{c, \text { Bazett }}$ and QT $\mathrm{C}_{\mathrm{c} \text { Hodges }}$ markers demonstrate nearperfect behavior on the receiver-operating characteristic (ROC) curve (Figure 2), which is reflected in the area under curve (AUC) value (Table 1). In contrast, both STAN-device analogue episodic and baseline T/QRS rise markers show AUC values close to random (0.50). This contrast is likewise visible in the degree of correlation between predicted and actual hypoxic/normoxic states. Interval-based HRV markers in the form of normalized low frequency (LF; 0.04-0.15 Hz) and high frequency (HF; $0.40-1.50 \mathrm{~Hz}$ ) band autospectral power and waveformbased episodic and baseline T/QRS rise markers show a weak correlation (Matthews correlation coefficient; MCC $\leq 0.35$ ) (12), whereas interval-based $\mathrm{HR}, \mathrm{PR} / \mathrm{RR}$ ratio, and both $\mathrm{HR}$-corrected QT markers were strongly correlated (MCC > 0.67).

\section{Identification of Hypoxic Individuals During UCO}

Low sensitivity does not directly imply that on the individual level hypoxia will not be detected. We tested the individual detection rates based on the assumption that a single positive result during the UCO period would constitute a detection of hypoxia. Accumulative detection of individuals during UCO is shown in Figure 3. The percentage of individuals with marker values indicating hypoxia at the 4-min mark, 12-min mark, and end of the UCO is shown in Supplementary Table S1 online. At the 4-min mark ( $\mathrm{pH}<7.1)$, hypoxia can be most clearly distinguished by assessing HR (96\% of individuals), PR/RR ratio (96\%), and Hodges' (91\%) and Bazett's corrected QT length (90\%). By 12 min of hypoxia ( $\mathrm{pH}<7.0$ ), these markers identify 97 to $99 \%$ of the fetuses as hypoxic. Waveform-based STAN episodic and baseline rise markers for hypoxia do not perform well. Sixty-five and $28 \%$ of the severely asphyxiated individuals were not detected by the end of the UCO using these markers.

\section{Baseline and Threshold Values}

An overview of population mean baseline values and positive and negative threshold values for predicting hypoxic/normoxic state is shown in Supplementary Table S2 online. Alarms were produced when the change with respect to the baseline value exceeded the positive threshold or decreased below the negative threshold. HR and PR/RR ratio markers only produced alarms when values decreased below the negative threshold. The STAN-device analogue episodic and baseline rise markers only produced alarms when values exceeded the positive threshold. Other markers produced alarms above positive and below negative threshold values. Notably, the T/QRS ratio marker produced $53 \%$ of its alarms when it exceeded the positive threshold and $47 \%$ of its alarms when it decreased below 
a

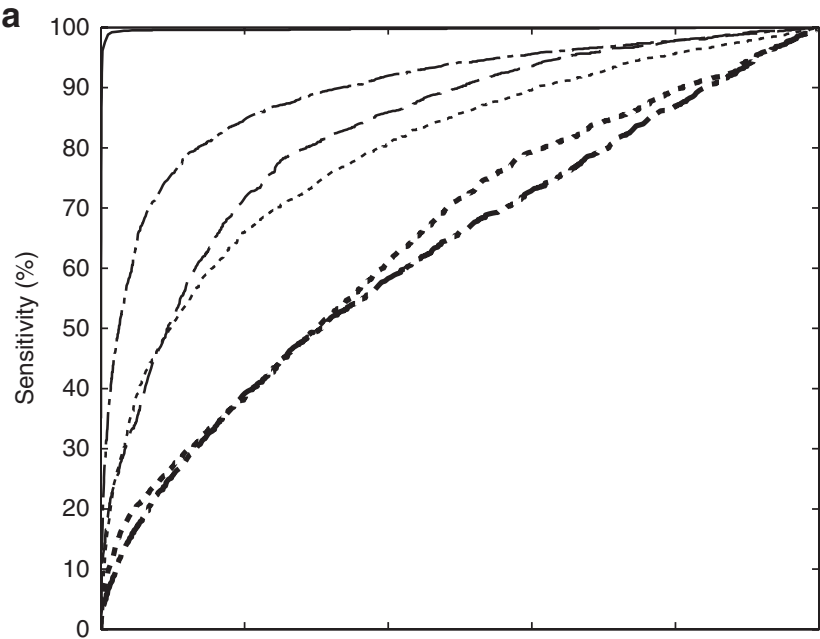

C

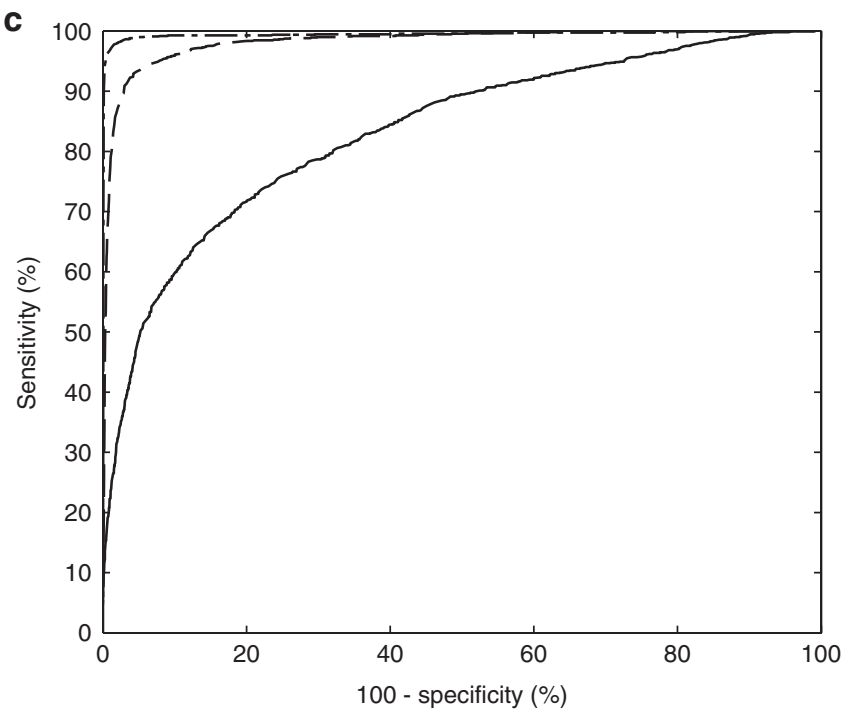

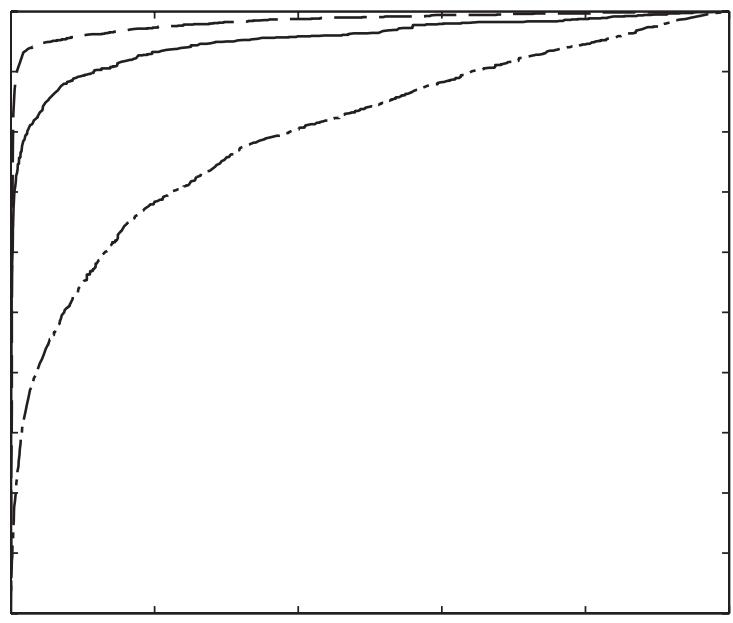

d

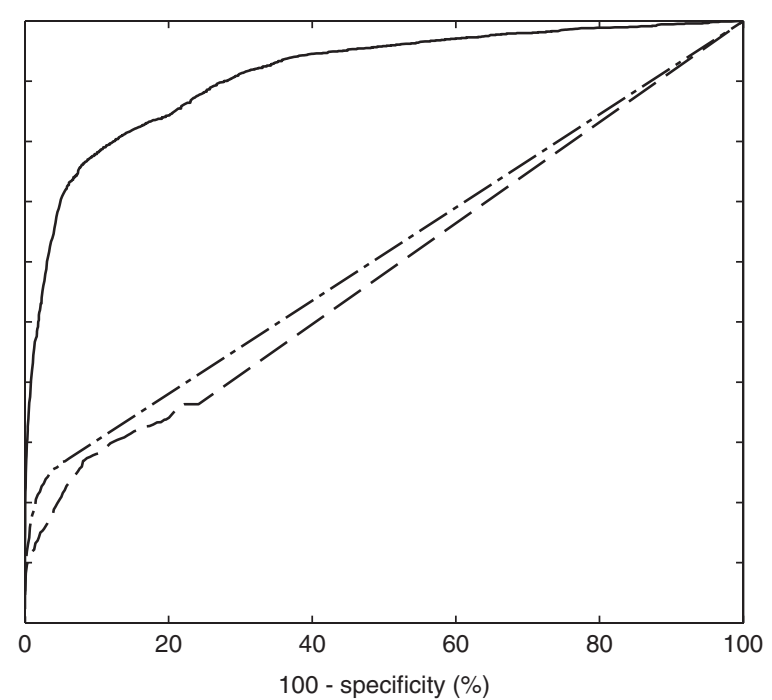

Figure 2. ROC curves for ECG markers for hypoxia. (a) Heart rate (solid line), heart rate variability (HRV; dashed line), low frequency (LF) HRV (thin dash-dot line), high frequency (HF) HRV (thin dotted line), normalized LF HRV (thick dash-dot line), and normalized HF HRV (thick dotted line). (b) PR interval (solid line), PR/RR ratio (dashed line), PR-HR correlation (dash-dot line). (c) QT (solid line), $\mathrm{QT}_{\mathrm{c}, \text { Bazett }}$ (dashed line), QT $\mathrm{C}_{\text {,Hodges }}$ (dash-dot line). (d) T/QRS ratio (solid line), STAN baseline rise (dashed line), STAN episodic rise (dash-dot line).

the negative threshold, in contrast to the STAN-device analogue markers that only produce alarms on positive changes.

\section{DISCUSSION}

Interval-based ECG markers generally performed better than waveform-based markers in detecting hypoxia in a standardized preterm ovine model for severe hypoxia-ischemia. The standardized period of complete UCO resulted in severe asphyxia which was characterized by acidosis. Hence, any potential clinically relevant marker was expected to deviate from baseline values at some point during UCO. Changes in interval-based HR, PR interval, PR/RR ratio, and Bazett's and Hodges' corrected QT correlated strongly with hypoxia. In contrast, waveform-based STAN episodic and baseline rise markers were only weakly correlated.

Decreases in fetal HR with respect to baseline were found to be sensitive and specific for hypoxia in our model. HR decelerations are a well known effect of hypoxia but can also be due to other factors, such as maternal movement, uterine contractions, and fetal sleep cycles, limiting specificity of the marker in clinical practice (1). Due to our experimental setup, the influence of nonhypoxic factors on HR was estimated to be less than within clinical settings, which led to near-perfect behavior based on the ROC curve.

Other interval-based markers were related to HR variability, both in the temporal and frequency domain. Depressed temporal HR variability correlates to acidosis, (13) and frequency domain-based normalized LF and HF HRV markers were likewise correlated to acidosis (14). Temporal HRV was calculated as the SD of HRs of $5 \mathrm{~min}$, and this is reflected in sharp transitions at the start and end of UCO (Supplementary Figure S3 online), when HR abruptly changes. The temporal HRV marker has a PPV of $38 \%$, which indicates that low temporal HRV also occurs during baseline period and not only during UCO. PPV might be improved by determining temporal HRV over longer periods of time, but at the cost of sensitivity and time to detection. 


\section{Articles | Zwanenburgetal.}

LF and HF HRV markers performed better than after their respective normalization by the total power in the $0.04-1.50$ $\mathrm{Hz}$ band of the RR interval autospectrum. This contrasts with the findings by van Laar et al. (14), who did not find significant hypoxia-induced changes in the LF and HF HRV markers but did find significant changes in their normalized counterparts. The different findings might be due to age of the subjects involved (term vs. preterm), which would indicate that domain-based HRV markers for hypoxia are age specific.

Changes in $\mathrm{PR} / \mathrm{RR}$ ratio were found to be highly correlated to hypoxia. However, the specificity of the PR/RR ratio as a marker for hypoxia and acidemia has been disputed (15). While the decrease in PR/RR ratio during UCO was clear, Westgate et al (15) concluded that under conditions of no or only mild acidosis a decrease in $\mathrm{PR} / \mathrm{RR}$ ratio was a marker of reflex responses to HR decelerations and that it did not provide additional information concerning the metabolic reserves of the fetus.

PR interval reflects atrioventricular delay and was found to strongly correlate with hypoxia. AV delay was generally increased during UCO (Supplementary Figure S4 online). Others have reported a decrease in AV delay due to hypoxia $(8,10)$. It is tempting to postulate that the different duration and number of hypoxic events are responsible for the observed differences. More precisely, a single long occlusion instead of a series of shorter occlusions was used in our study to induce hypoxia and acidosis. Atrioventricular delay may be increased due to severely depleted metabolic reserves during the single occlusion. Because the PR marker seems to depend on the precise course of hypoxia, its clinical effectiveness is questionable. A similar observation can be made with regard to PR-HR correlation. For the PR-HR correlation marker, 64 and 36\% of alarms for hypoxia were due to respectively an increase above the positive threshold or a decrease below the negative threshold. Therefore, PR and PR-HR correlation are unlikely to be clinically effective markers, which is corroborated by the inconclusive results with regard to these markers found in the clinical trial by Strachan et al (16).

We assessed Hodges' and Bazett's corrected QT markers because the former is least correlated with HR (17) and the latter is a commonly used correction to the QT interval. Changes in Hodges' and Bazett's corrected QT markers were found to be very sensitive and specific to hypoxia and demonstrated high PPV as well. QT interval length was stable during the baseline period and decreased during hypoxia. This finding is supported by the observations of Oudijk et al. (11), who demonstrated that $\mathrm{QT}_{\mathrm{c}, \text { Bazett }}$ interval shortening did not depend on HR and was unrelated to normal labor stress. QT shortening may be linked to a catecholamine surge that is released when energy balance in the myocardium becomes negative due to hypoxia and anaerobic myocardial glycolysis starts $(11,18-20)$. The HR-corrected QT interval markers may therefore be useful as specific markers for hypoxia.

Because clinical guidelines for STAN are specified for infants older than $36 \mathrm{wk}$ of gestation, it can be argued that STAN is
Table 1. Classification performance of individual ECG markers for hypoxia

\begin{tabular}{lcccccc}
\hline ECG marker & Sensitivity & Specificity & PPV & NPV & MCC & AUC \\
\hline Heart rate & $\mathbf{0 . 9 6}$ & $\mathbf{1 . 0 0}$ & $\mathbf{0 . 9 8}$ & $\mathbf{0 . 9 9}$ & $\mathbf{0 . 9 7}$ & $\mathbf{0 . 9 9}$ \\
HRV & 0.58 & 0.88 & 0.38 & $\mathbf{0 . 9 4}$ & 0.38 & 0.82 \\
LF HRV & 0.66 & $\mathbf{0 . 9 5}$ & 0.64 & $\mathbf{0 . 9 5}$ & 0.60 & 0.89 \\
HF HRV & 0.40 & $\mathbf{0 . 9 5}$ & 0.50 & 0.92 & 0.39 & 0.79 \\
Normalized LF HRV & 0.22 & $\mathbf{0 . 9 2}$ & 0.28 & $\mathbf{0 . 9 0}$ & 0.16 & 0.62 \\
Normalized HF HRV & 0.17 & $\mathbf{0 . 9 7}$ & 0.43 & $\mathbf{0 . 9 0}$ & 0.21 & 0.65 \\
PR interval & 0.75 & $\mathbf{0 . 9 9}$ & $\mathbf{0 . 9 1}$ & $\mathbf{0 . 9 7}$ & 0.80 & $\mathbf{0 . 9 5}$ \\
PR/RR ratio & $\mathbf{0 . 9 0}$ & $\mathbf{0 . 9 9}$ & $\mathbf{0 . 9 3}$ & $\mathbf{0 . 9 9}$ & $\mathbf{0 . 9 1}$ & $\mathbf{0 . 9 7}$ \\
PR-HR correlation & 0.37 & $\mathbf{0 . 9 7}$ & 0.64 & $\mathbf{0 . 9 2}$ & 0.44 & 0.79 \\
QT $_{\text {uncorrected }}$ & 0.50 & $\mathbf{0 . 9 5}$ & 0.53 & $\mathbf{0 . 9 4}$ & 0.46 & 0.83 \\
QTC $_{\text {Bazett }}$ & 0.86 & $\mathbf{0 . 9 8}$ & 0.86 & $\mathbf{0 . 9 8}$ & 0.84 & $\mathbf{0 . 9 7}$ \\
QTC $_{\text {Hodges }}$ & $\mathbf{0 . 9 4}$ & $\mathbf{1 . 0 0}$ & $\mathbf{0 . 9 8}$ & $\mathbf{0 . 9 9}$ & $\mathbf{0 . 9 6}$ & $\mathbf{0 . 9 9}$ \\
T/QRS ratio $_{\text {STAN episodic rise }}$ & 0.70 & $\mathbf{0 . 9 5}$ & 0.65 & $\mathbf{0 . 9 6}$ & 0.63 & $\mathbf{0 . 9 1}$ \\
STAN baseline rise $^{\mathrm{a}}$ & 0.10 & $\mathbf{1 . 0 0}$ & 0.84 & 0.88 & 0.26 & 0.58 \\
\hline
\end{tabular}

Performance values above 0.90 are emphasized using boldface.

AUC, area under curve; $H F$, high frequency; HRV, heart rate variability; LF, low frequency; MCC, Matthews correlation coefficient; NPV, negative predictive value; PPV, positive predictive value; STAN, ST-segment analysis.

${ }^{a}$ Marker is not corrected for individual baseline, by definition.

not applicable to a preterm ovine model, such as the one used here. However, it was demonstrated that the response of the ST waveform to hypoxia is similar in preterm and term ovine fetuses $(21,22)$ and thus that ST waveform analysis can be reliably performed even in the preterm fetus.

However, STAN-device analogue episodic and baseline rise markers did not differ from normoxic baseline values in 65 and $28 \%$ of the fetuses at any point during UCO. The negligible correlation and low sensitivity for these markers indicate that these markers cannot be used to distinguish between hypoxia and normoxia. Our analysis did not provide evidence to support the hypothesis that STAN episodic and baseline markers are clinically useful. Whereas UCO in our model resulted in severe hypoxia and acidemia, in clinical practice, perinatal hypoxia is commonly manifested through multiple shorter periods of hypoxia and is usually less severe. The fact that STAN episodic and baseline rise markers performed poorly under severe hypoxic conditions makes better performance under less severe conditions unlikely.

Waveform-based STAN episodic and baseline rise markers have already been implemented in a clinical device. The poor performance of these markers is thus surprising, yet supported by meta-analyses of clinical trials $(5,6)$. As the name suggests, STAN episodic and baseline rise markers assume that hypoxia induces a rise in T/QRS ratio. In our model, several subjects responded to hypoxia by developing more negative $\mathrm{T}$ waves (Supplementary Figure S5 online). This is ignored by focusing solely on positive T/QRS ratio changes. 
a

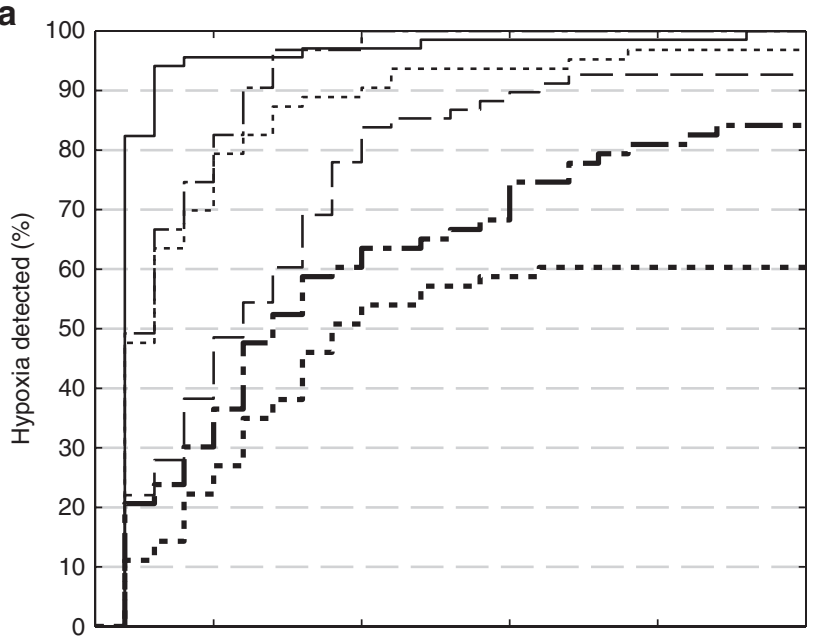

C

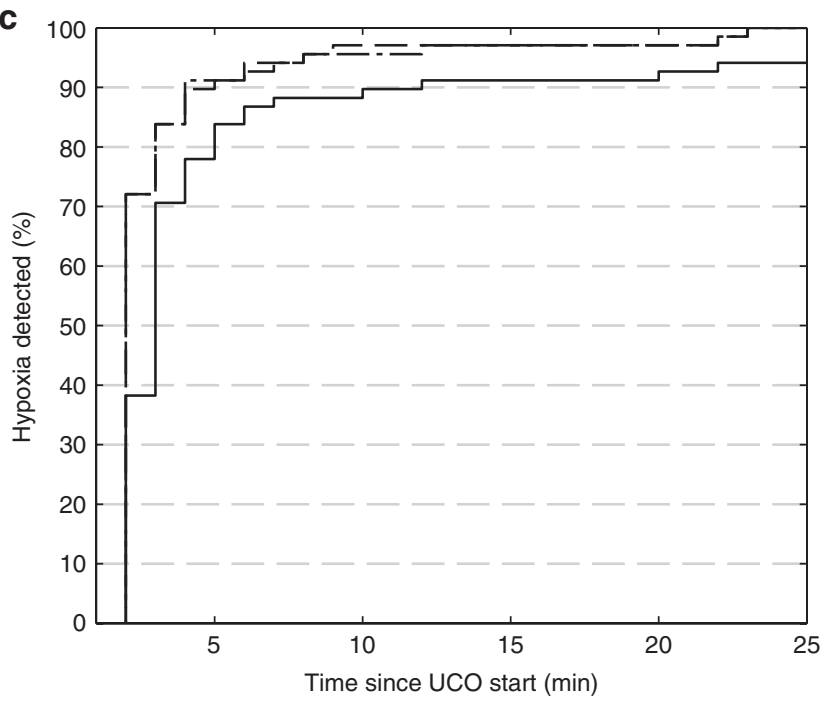

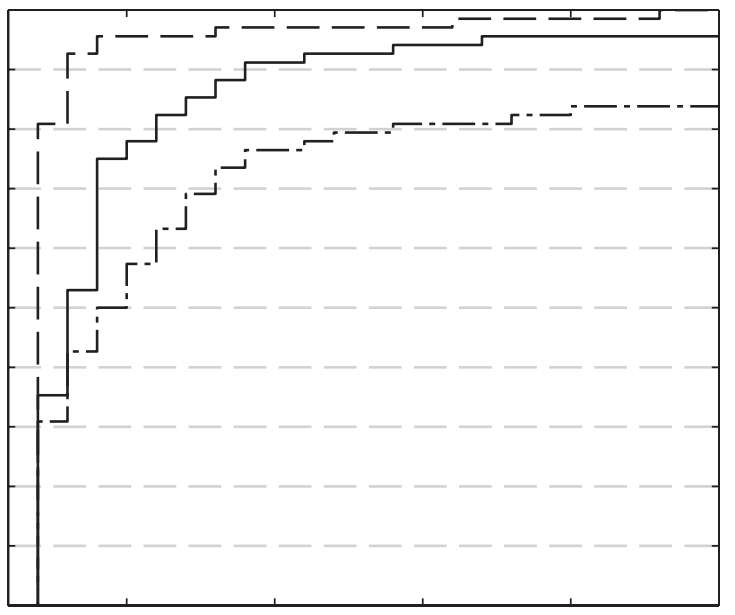

d

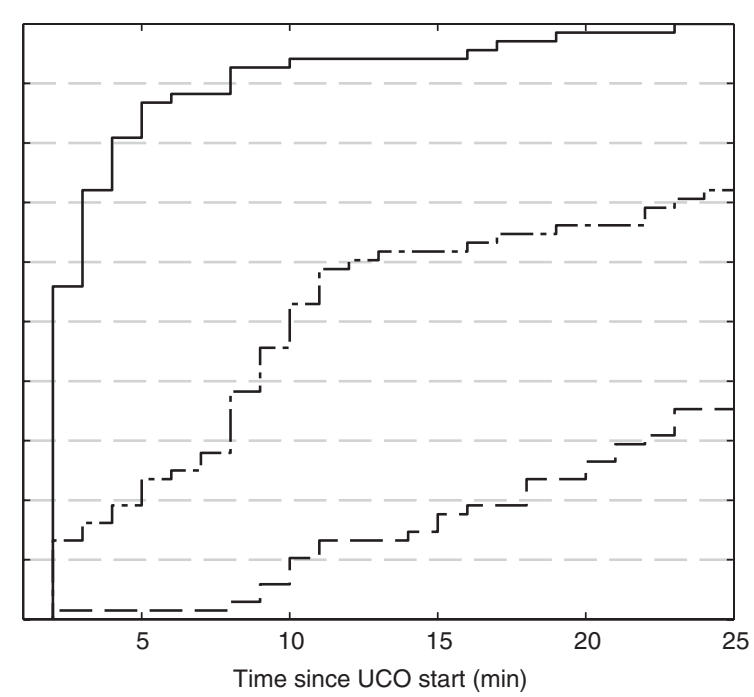

Figure 3. Detection plots for the percentage of individuals where hypoxia was detected during UCO. (a) Heart rate (solid line), heart rate variability (HRV; dashed line), low frequency (LF) HRV (thin dash-dot line), high frequency (HF) HRV (thin dotted line), normalized LF HRV (thick dash-dot line), and normalized HF HRV (thick dotted line). (b) PR interval (solid line), PR/RR ratio (dashed line), PR-HR correlation (dash-dot line). (c) QT (solid line), QT ${ }_{c, B a z e t t}$ (dashed line), $\mathrm{QT}_{\text {c.Hodges }}$ (dash-dot line). (d) T/QRS ratio (solid line), STAN baseline rise (dashed line), STAN episodic rise (dash-dot line).

A general disadvantage of waveform-based markers is that the shape of the ECG waveform depends on the orientation of the ECG lead with respect to the heart vector (Figure 1). Differences in lead orientation, differences in cardiac anatomy, and differences in cardiac response to hypoxia between individuals cause variance in waveform-based marker values. Interval-based markers do not rely directly on the shape of ECG waveforms. Therefore, interval-based markers are hypothesized to provide more stable performance than waveform-based markers.

As mentioned above, fetuses in this study were exposed to profound hypoxia-ischemia. Because profound hypoxia-ischemia should produce a clear change in any potential marker for hypoxia, our model allowed evaluation and comparison of markers to identify potential markers for hypoxia. Generally, the clinical onset of hypoxia is gradual. In clinical cases, the baseline period is harder to define, and HR patterns are far less predictive (2). We expect that marker performance in clinical cases will be lower than that reported here.

\section{Conclusion}

The most suitable ECG markers for detecting fetal hypoxia were interval-based, rather than waveform-based. Intervalbased markers HR and HR-corrected QT length were able to detect fetal hypoxia in our standardized ovine model and constitute promising markers for detecting hypoxia in human fetuses. In contrast, waveform-based STAN episodic and baseline T/QRS rise markers, which were our analogue of the markers implemented in clinical STAN devices, could not assess severe hypoxia-ischemia.

\section{METHODS}

\section{Animals}

The study group for this retrospective study consisted of 74 fetuses of both genders of time-mated Texel ewes, measured in the period from February 2010 to November 2013. Five animals were excluded due to incomplete UCOs. The study group was originally set up to investigate processes and treatment of preterm hypoxic-ischemic encephalopathy (23-25). The original studies were approved by the local Animal Ethics Research Committee (DEC-UM, Maastricht 
Filtered ECG $(0.05-100 \mathrm{~Hz})$

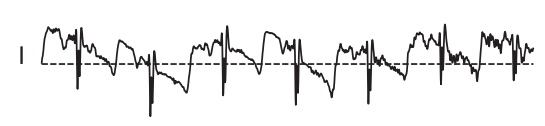

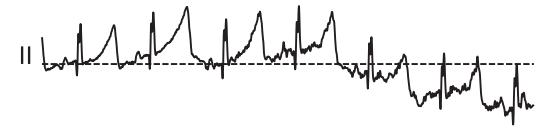

b

Wavelet baseline correction

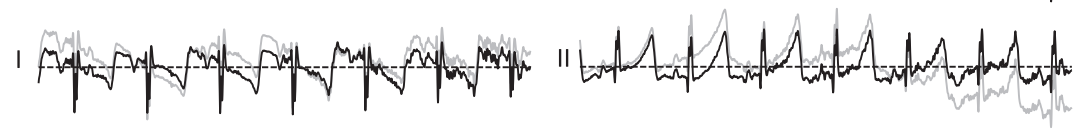

c

Lead projection

I \& II $\rightarrow-$ aVF

d

Q wave top detection

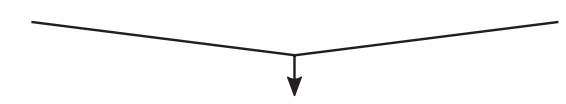

Linear baseline correction

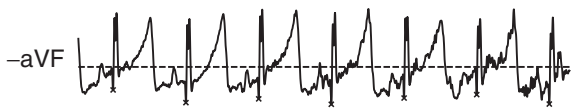

f
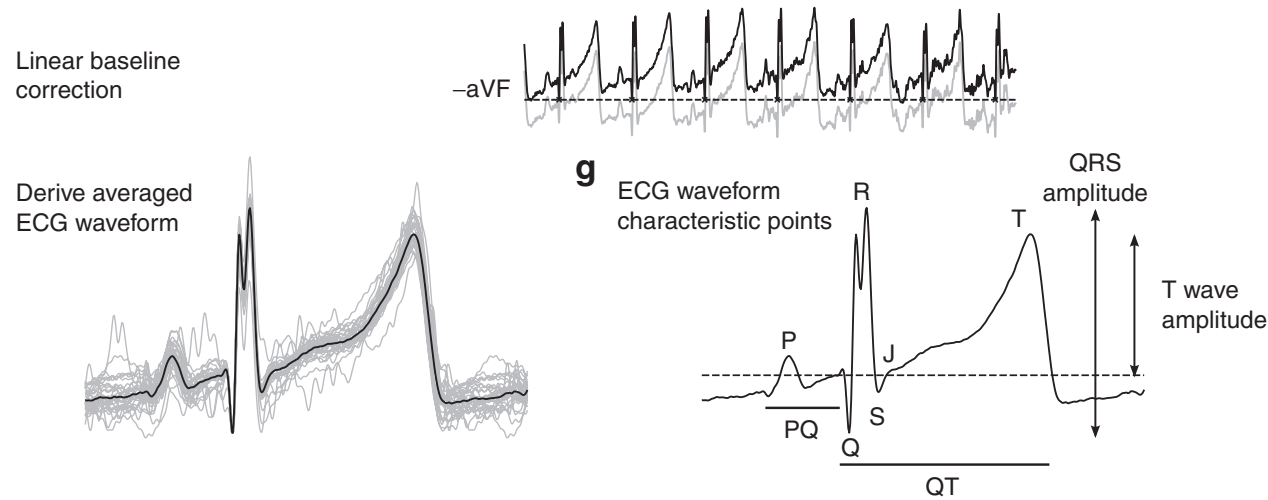

Figure 4. Illustration of ECG pre-processing steps. (a) ECG recordings for leads I and II are first filtered using a Butterworth filter. (b) Subsequently, the filtered signal (gray) is decomposed using a wavelet and partially reconstructed (black) to suppress baseline wander. (c) ECG leads I and II are digitally projected to a negative aVF lead. (d) Q wave tops (black cross) are detected in the -aVF lead. (e) Amplitude differences between Q wave tops are linearly interpolated and used for further correction of baseline wander of the ECG. (f) Thirty truncated ECG waveforms are aligned on the detected Q wave top and averaged. (g) Characteristic points, intervals, and waves of the ECG. T amplitude is the amplitude of the T wave above baseline and QRS amplitude is the largest of QR and RS amplitudes.

University, Maastricht, the Netherlands; approval reference numbers: DEC 2010-131, DEC 2012-064) and conducted at the Maastricht University Medical Center, Maastricht, the Netherlands.

\section{Surgical Procedure}

Fetuses were instrumented at $101.5 \pm 1.2$ (mean \pm SD) d of gestational age (GA; full gestation equals $146 \mathrm{~d})$. The surgical procedure was described previously (24). Ewes received intravenous prophylactic antibiotics (1,000 mg amoxicillin and $200 \mathrm{mg}$ clavulanic acid). Anesthesia was induced by i.v. administered thiopenthal $(15 \mathrm{mg} / \mathrm{kg})$. After intubation, general anesthesia was maintained with 1 to $2 \%$ isoflurane guided by depth of sedation and supplemented by remifentanyl i.v. ( $0.75 \mu \mathrm{g} / \mathrm{kg} / \mathrm{min})$ for analgesia. During surgery, vital parameters and depth of sedation were continuously monitored by certified personnel. A catheter was placed in the maternal long saphenous vein to provide access for a perioperative saline drip $(250 \mathrm{ml} / \mathrm{h})$ and for postoperative blood sampling and administration of prophylactic antibiotics for $4 \mathrm{~d}$.

The fetus was exposed through a lower midline laparotomy. Two catheters (3.5 French polyurethane umbilical vessel catheters; Tyco Healthcare Group, Mansfield, MA) were inserted in the brachial vein and femoral artery. A vascular occluder (OC16HD, In Vivo Metric, Healdsburg, CA) was placed around the umbilical cord. Three silver electrodes ( $5 \mathrm{~mm}$; Cooner Wire, Chatsworth, CA) were fixed s.c. with sutures for continuous fetal ECG recording, forming ECG leads I, II, and III. After repositioning the fetus, an amniotic catheter was inserted into the amniotic cavity and used to fill the cavity with warm sterile saline and $80 \mathrm{mg}$ of Gentamycin. The electrode leads and catheters were exteriorized through the flank of the ewe, and incisions were sutured. After surgery, catheters were continuously perfused with heparinized saline $(25 \mathrm{IU} / \mathrm{ml}, 0.2 \mathrm{ml} / \mathrm{h})$. The ewe and fetus were allowed to recover from surgery for 3 to $4 \mathrm{~d}$. At $105.6 \pm 1.0$ (mean \pm SD) days, the umbilical cord was occluded for 25 min by rapidly inflating the occluder with sterile saline. Blood pressure and HR were monitored, and intermittent blood gas measurements were performed before, during, and after UCO.

Animals were housed together when possible and had ad libitum access to water and food. Surgical wounds were inspected daily and treated with chlortetracycline spray to prevent infection. The welfare of the animals was monitored daily by certified personnel. The animals were maintained for $7 \mathrm{~d}$ after occlusion. At this point, both ewe and fetus were killed by i.v. administration of pentobarbital $(200 \mathrm{mg} / \mathrm{kg})$.

\section{Data Acquisition}

ECG data were acquired and digitized by an MPAQ unit (MaastrichtProgrammable AcQuisition System; Maastricht Instruments, Maastricht, The Netherlands) (26) at a sample frequency of $250 \mathrm{~Hz}$ in the first 12 fetuses or, after update of the AD-converter, $1,000 \mathrm{~Hz}$ in the remaining 57 fetuses. ECG data were analyzed from $4 \mathrm{~h}$ before UCO until $4 \mathrm{~h}$ after occlusion.

\section{Data Processing}

All data processing and analysis was automatically performed using custom scripts in Matlab 2014a (The MathWorks, Natick, MA). The first step of the analysis was pre-processing ECG data for each fetus to derive averaged ECG waveforms $\left(\mathrm{ECG}_{\mathrm{avg}}\right)$ for every 30 beats. The preprocessing steps are illustrated in Figure 4 . In short, ECG was filtered using a $0.05-100 \mathrm{~Hz}$ third-order bidirectional Butterworth bandpass filter and a $50 \mathrm{~Hz}$ third-order bidirectional infinite impulse response notch filter to reduce signal drift, high frequency noise, and mains noise (Figure 4a). To remove major baseline deviations, the ECG was subsequently decomposed using a Daubechies 5-wavelet, up to the 6 th $(250 \mathrm{~Hz})$ or 8 th $(1,000 \mathrm{~Hz})$ level $(27)$. The residual was regarded as a baseline deviation and subtracted from the ECG signal (Figure $4 \mathrm{~b}$ ). 


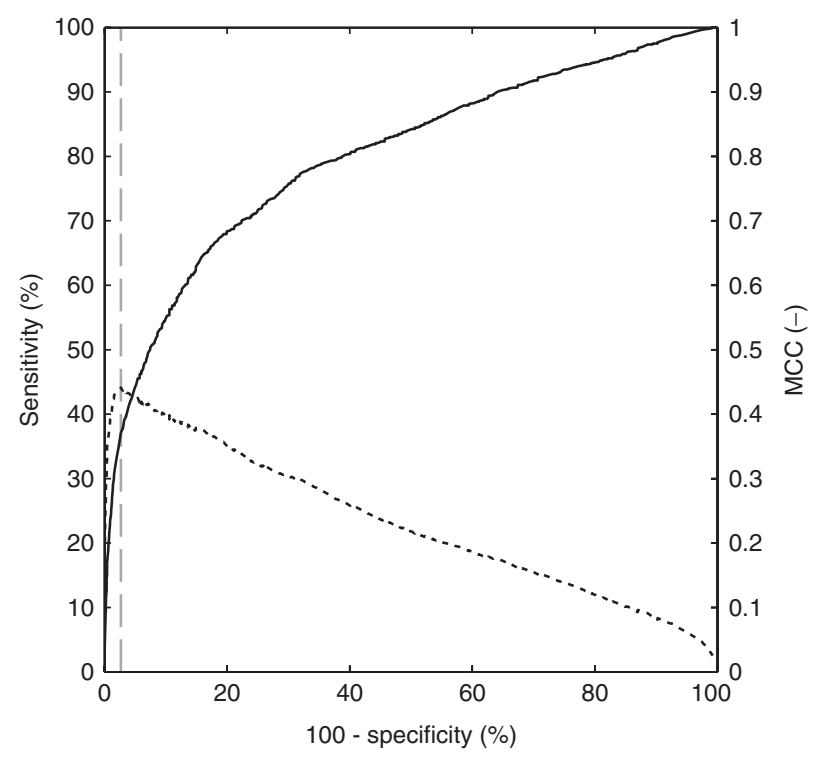

Figure 5. ROC curve (solid line) for T/QRS ratio and its corresponding Matthews correlation coefficient (MCC; dotted line). The vertical gray dashed line intersects the ROC curve at maximum MCC.

To imitate the unipolar scalp electrode used to record invasive ECG for ST waveform analyses, leads I and II were digitally projected to a negative aVF lead (Figure 4c). To determine cycle length (CL), and to define a suitable feature for waveform averaging, we determined the position of the $\mathrm{Q}$ wave top in the -aVF lead using our adaptation of the Pan Tompkins algorithm (28) (Figure 4d). Q wave top was used instead of $R$ wave top because the $R$ wave was split in all fetuses. The split $\mathrm{R}$ wave tops varied in amplitude and position, and therefore did not represent a stable feature in the ECG. Differences between vertical positions of subsequent $\mathrm{Q}$ wave tops were linearly interpolated and subtracted, thus aligning each $\mathrm{Q}$ wave top with 0 (Figure 4e) (29). To derive $\mathrm{ECG}_{\mathrm{avg}}$, we set the 25th percentile of cycle lengths from 30 subsequent individual ECG waveforms (ECG as $_{\text {and }}$ ) as CL25. Each of the $30 \mathrm{ECG}_{\text {ind }}$ waveforms was truncated in the interval $\left(\mathrm{Q}-0.4^{*} \mathrm{CL} 25, \mathrm{Q}+0.8{ }^{*} \mathrm{CL} 25\right)$. This interval was chosen to capture relevant features and intervals of the ECG waveform. The truncated waveforms were averaged by omitting $30 \%$ of the outermost values at each sample point and calculating the mean over the remaining $70 \%$ (Figure 4f). Occasionally, ECG thus derived should be omitted due to low signal quality of part of the $30 \mathrm{ECG}_{\text {ind }}$ from which ECG was derived. Quality of ECG $\mathrm{G}_{\text {avg }}$ was verified by deriving a signalto-noise ratio. This signal-to-noise ratio was defined as the base-10 logarithm of the squared length of $\mathrm{ECG}_{\mathrm{avg}}-$ a surrogate for true signal variance-divided by the mean squared length of the corresponding $30 \mathrm{ECG}_{\text {ind }}$ - a surrogate for noise-induced variance. Waveforms with signal-to-noise ratio below the empirically established threshold of -0.25 were removed from further analysis.

\section{ECG-Based Physiological Markers}

Figure $4 \mathrm{~g}$ illustrates characteristic points, segments, and intervals of the ECG. $\mathrm{Q}$ wave onset, $\mathrm{P}$ wave onset, $\mathrm{R}$ wave top, $\mathrm{S}$ wave top, J point, and $T$ wave end were detected in ECG ${ }_{\text {avg }}$ using our adaptation of the Pan Tompkins algorithm. Q wave onset and P wave onset were also detected in ECG $_{\text {ind }}$. Q wave onset was used as ECG baseline, since at high HRs, the T wave partially overlaps and merges with the $\mathrm{P}$ wave, thereby slanting the PQ segment commonly used to define the ECG baseline.

Values for all ECG markers were updated every 30 beats, i.e., at every subsequent derivation of $\mathrm{ECG}_{\mathrm{avg}}$. The values of the various markers were calculated as follows.

$\mathrm{HR}$ was determined by calculation of the mean $\mathrm{HR}$ of the 30 most recent $\mathrm{ECG}_{\text {ind }}(30)$. HR variability was calculated by determining the $\mathrm{SD}$ of HRs of $\mathrm{ECG}_{\text {ind }}$ in the previous $5 \mathrm{~min}$ (30). Frequency domain

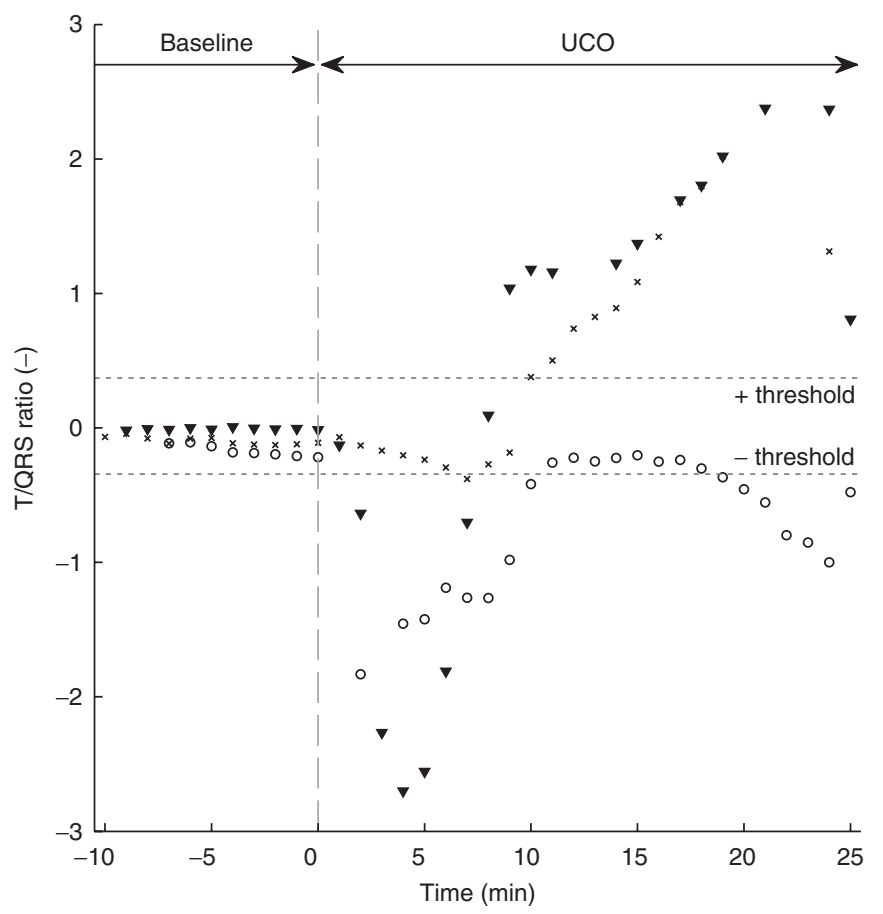

Figure 6. Thresholds for changes in T/QRS ratio with respect to individual baseline. T/QRS values of three subjects are shown (black cross: animal 1; black triangle: animal 2; open circle: animal 3). To clearly show T/QRS values during UCO only part of the baseline is shown. An alarm for hypoxia was given if the change in T/QRS ratio was above the positive threshold (+; $\mathrm{T} / \mathrm{QRS}=0.37)$ or was below the negative $(-; \mathrm{T} / \mathrm{QRS}=-0.34)$ threshold.

markers for HR variability were calculated as defined by van Laar et al. (14) Specifically, spectral power was calculated in the LF $(0.04-0.15$ $\mathrm{Hz})$ and $\mathrm{HF}(0.40-1.50 \mathrm{~Hz})$ bands of the RR interval autospectrum for RR intervals in the previous $64 \mathrm{~s}$. Next to LF and HF band spectral power, normalized power was calculated by dividing the LF and HF band spectral power by the total spectral power in the $0.04-1.50 \mathrm{~Hz}$ band.

Atrioventricular conduction delay was estimated by the length of the PR interval. The PR interval was defined as the interval between the onset of the $\mathrm{P}$ wave and the start of the QRS complex. The PR/RR ratio is calculated for by dividing the PR interval by the RR interval for the 30 most recent ECG and calculating their mean value. We used the approach of van Wijngaarden et al. (9) to assess the PR-HR relationship and calculated the Pearson correlation coefficient between PR intervals and HRs of ECG ind $_{\text {in }}$ the previous $150 \mathrm{~s}$.

QT interval length represents ventricular activation and repolarization time. The QT interval was defined as the time interval between the onset of the $\mathrm{Q}$ wave and the end of the $\mathrm{T}$ wave in $\mathrm{ECG}_{\mathrm{avg}}$. QT interval length decreases with increasing $\mathrm{HR}$ and is therefore corrected for $\mathrm{HR}$ to reduce $\mathrm{HR}$ dependency. In addition to Hodges' correction $\left(\mathrm{QT}_{\mathrm{c} \text { todges }}=\mathrm{QT}+1.75(\mathrm{HR}-60)\right)$ and Bazett's correction $\left(\mathrm{QT}_{c, \text { Bazett }}=\mathrm{QT}(\mathrm{HR} / 60)^{1 / 2}\right)$, we also assessed the uncorrected QT interval.

T/QRS ratio was calculated as the maximal positive or negative deflection between S wave top or J point-when present-and the end of the T wave, divided by the amplitude of the QRS complex in ECG (Figure 4g). The clinically used and commercially available STAN device assesses episodic and baseline rise in T/QRS values as ECG markers for hypoxia. Analogue to STAN device alarms, episodic rise in T/QRS value was defined as a positive change between the actual $\mathrm{T} / \mathrm{QRS}$ value and the median T/QRS value of the previous $10 \mathrm{~min}$ (4). Also analogue to STAN device alarms, baseline rise in T/QRS value was defined as a positive change from baseline by comparison of the median $\mathrm{T} / \mathrm{QRS}$ value of the last $10 \mathrm{~min}$ with the baseline T/QRS value (4). The baseline T/QRS value was defined as the lowest nonnegative 
median T/QRS value in a 20 -min moving window within the previous $3 \mathrm{~h}$ of recording.

\section{Blood Gasses}

We sampled fetal arterial blood to obtain baseline values for blood acidity $(\mathrm{pH})$, arterial partial oxygen pressure $\left(\mathrm{PaO}_{2}\right)$, and arterial partial carbon dioxide pressure $\left(\mathrm{PaCO}_{2}\right)$. Samples for the baseline period were drawn $1 \mathrm{~h}$ before UCO took place. During UCO, samples were withdrawn at $5,10,15$, and 20 min after UCO start. In the recovery period, samples were drawn at 3,10,20,30, and 60 min after UCO ended.

\section{Performance Analysis}

Interval- and waveform-based ECG markers were grouped within 1-min windows as follows to facilitate comparison between markers. Markers within each 1-min window were represented by the median value, except for STAN episodic and baseline rise markers, which were represented by the maximum value within the 1-min window. We based performance analysis on changes in marker values with respect to the baseline in the individual fetus. We defined the individual baseline as the mean value of the marker during the baseline period for the fetus under consideration. STAN episodic or baseline rise markers were not corrected for the individual baseline, as they were already corrected for baseline in a different way. Subsequently, the individual baseline value was subtracted from windowed marker values to determine (normalized) changes with respect to baseline.

Area under the ROC curve (AUC) is a general measure for the ability of an ECG marker to differentiate between hypoxic and normoxic states. To construct ROC curves, normalized values during the baseline and UCO period were first sorted. Then, detection thresholds for positive and negative changes were iteratively increased. A contingency table was calculated for each combination of positive and negative thresholds. Sensitivity and specificity were calculated from these contingency tables for the purpose of creating the ROC curve. Subsequently AUC was calculated.

MCC $(31,32)$ was calculated for each of the contingency tables underlying the ROC curve to define the optimal correlation between actual and predicted hypoxic/normoxic states. The positive and negative thresholds corresponding to the optimal correlation were used to define sensitivity, specificity, PPV, and negative predictive values (Figures 5 and 6).

Performance analysis using ROC disregards the temporal behavior of ECG markers during UCO. To assess the ability of ECG-based markers to distinguish between a normoxic and hypoxic state on the individual level, we constructed cumulative detection curves. The detection curves were subsequently used to tabulate the percentage of detected individuals at several time points during UCO. The percentage of detected individuals was assessed at $4 \mathrm{~min}$ (onset of metabolic acidosis; $\mathrm{pH}<7.1(33,34)), 12$ minutes (progressive acidosis; $\mathrm{pH}<7.0)(35)$, and at the end of the UCO at $25 \mathrm{~min}$.

\section{SUPPLEMENTARY MATERIAL}

Supplementary material is linked to the online version of the paper at http:// www.nature.com/pr

\section{ACKNOWLEDGMENTS}

The authors acknowledge the excellent support received from Dennis Kruk, Elke Kuypers, Maria Nikifourou, and Monique Willems during surgical procedures and by monitoring animal well-being.

\section{STATEMENT OF FINANCIAL SUPPORT}

This current work was supported by EURON School of Mental Health and Neuroscience, Maastricht University, Maastricht, The Netherlands.

Disclosure: R.V. is scientific director and shareholder of Nemo Healthcare BV. The other authors declare no conflict of interest.

\section{REFERENCES}

1. Alfirevic Z, Devane D, Gyte GML. Continuous cardiotocography (CTG) as a form of electronic fetal monitoring (EFM) for fetal assessment during labour. Cochrane Libr 2013;3:CD006066.

2. Larma JD, Silva AM, Holcroft CJ, Thompson RE, Donohue PK, Graham EM. Intrapartum electronic fetal heart rate monitoring and the identi- fication of metabolic acidosis and hypoxic-ischemic encephalopathy. Am J Obstet Gynecol 2007;197:301.e1-8.

3. Belfort MA, Saade GR. ST segment analysis as an adjunct to electronic fetal monitoring. Part I: background, physiology, and interpretation. Clin Perinatol 2011;38:143-57, vii.

4. Amer-Wåhlin I, Yli B, Arulkumaran S. Foetal ECG and STAN technology-a review. Eur Clin Obstet Gynaecol 2005;1:61-73.

5. Neilson JP. Fetal electrocardiogram (ECG) for fetal monitoring during labour. Cochrane Database Syst Rev 2013;3:CD000116.

6. Salmelin A, Wiklund I, Bottinga R, et al. Fetal monitoring with computerized ST analysis during labor: a systematic review and meta-analysis. Acta Obstet Gynecol Scand 2013;92:28-39.

7. Widmark C, Lindecrantz K, Murray H, Rosén KG. Changes in the PR, RR intervals and ST waveform of the fetal lamb electrocardiogram with acute hypoxemia. J Dev Physiol 1992;18:99-103.

8. Westgate JA, Gunn AJ, Bennet L, Gunning MI, de Haan HH, Gluckman PD. Do fetal electrocardiogram PR-RR changes reflect progressive asphyxia after repeated umbilical cord occlusion in fetal sheep? Pediatr Res 1998;44:297-303.

9. van Wijngaarden WJ, de Haan HH, Sahota DS, James DK, Symonds EM, Hasaart TH. Changes in the PR interval-fetal heart rate relationship of the electrocardiogram during fetal compromise in chronically instrumented sheep. Am J Obstet Gynecol 1996;175:548-54.

10. Keunen H, van Wijngaarden WJ, Sahota DS, Hasaart TH. The PR intervalfetal heart rate relationship during repetitive umbilical cord occlusions in immature fetal sheep. Eur J Obstet Gynecol Reprod Biol 2000;89:69-74.

11. Oudijk MA, Kwee A, Visser GH, Blad S, Meijboom EJ, Rosén KG. The effects of intrapartum hypoxia on the fetal QT interval. BJOG 2004;111:656-60.

12. Taylor R. Interpretation of the correlation coefficient: a basic review. J Diagn Med Sonogr 1990;6:35-9.

13. Parer JT, King T, Flanders S, Fox M, Kilpatrick SJ. Fetal acidemia and electronic fetal heart rate patterns: is there evidence of an association? J Matern Fetal Neonatal Med 2006;19:289-94.

14. van Laar JO, Peters CH, Vullings R, Houterman S, Bergmans JW, Oei SG. Fetal autonomic response to severe acidaemia during labour. BJOG 2010;117:429-37.

15. Westgate JA, Bennet L, Gunn AJ. The role of fetal ECG monitoring in labour. Fetal Matern Med Rev 2002;13:119-39.

16. Strachan BK, van Wijngaarden WJ, Sahota D, Chang A, James DK. Cardiotocography only versus cardiotocography plus PR-interval analysis in intrapartum surveillance: a randomised, multicentre trial. FECG Study Group. Lancet 2000;355:456-9.

17. Luo S, Michler K, Johnston P, Macfarlane PW. A comparison of commonly used QT correction formulae: the effect of heart rate on the QTc of normal ECGs. J Electrocardiol 2004;37:Suppl:81-90.

18. Rosén KG, Dagbjartsson A, Henriksson BA, Lagercrantz H, Kjellmer I. The relationship between circulating catecholamines and ST waveform in the fetal lamb electrocardiogram during hypoxia. Am J Obstet Gynecol 1984;149:190-5.

19. Algra A, Roelandt JR, Tijssen JG, Simoons ML, Pool J. Effect of beta-blockers on the relation between QT-interval and heart rate in exercise ECG. Eur Heart J 1987;8:Suppl D:71-3.

20. Abildskov JA. Adrenergic effects of the QT interval of the electrocardiogram. Am Heart J 1976;92:210-6.

21. Wassink G, Galinsky R, Drury PP, Gunn ER, Bennet L, Gunn AJ. Does maturity affect cephalic perfusion and T/QRS ratio during prolonged umbilical cord occlusion in fetal sheep? Obstet Gynecol Int 2014;2014:314159.

22. Welin AK, Blad S, Hagberg H, Rosén KG, Kjellmer I, Mallard C. Electrocardiographic changes following umbilical cord occlusion in the midgestation fetal sheep. Acta Obstet Gynecol Scand 2005;84:122-8.

23. Jellema RK, Lima Passos V, Ophelders DR, et al. Systemic G-CSF attenuates cerebral inflammation and hypomyelination but does not reduce seizure burden in preterm sheep exposed to global hypoxia-ischemia. Exp Neurol 2013;250:293-303.

24. Jellema RK, Lima Passos V, Zwanenburg A, et al. Cerebral inflammation and mobilization of the peripheral immune system following global hypoxia-ischemia in preterm sheep. J Neuroinflammation 2013; 10:13. 
25. Jellema RK, Wolfs TG, Lima Passos V, et al. Mesenchymal stem cells induce $\mathrm{T}$-cell tolerance and protect the preterm brain after global hypoxiaischemia. PLoS One 2013;8:e73031.

26. Zwanenburg A, Jellema RK, Jennekens W, et al. Heart rate-mediated blood pressure control in preterm fetal sheep under normal and hypoxicischemic conditions. Pediatr Res 2013;73:420-6.

27. Zhang D. Wavelet approach for ECG baseline wander correction and noise reduction. Conf Proc IEEE Eng Med Biol Soc 2005;2:1212-5.

28. Pan J, Tompkins WJ. A real-time QRS detection algorithm. IEEE Trans Biomed Eng 1985;32:230-6.

29. Boucheham B, Ferdi Y, Batouche MC. Piecewise linear correction of ECG baseline wander: a curve simplification approach. Comput Methods Programs Biomed 2005;78:1-10.

30. Malik M, Bigger JT, Camm AJ, et al. Heart rate variability standards of measurement, physiological interpretation, and clinical use. Eur Heart J 1996;17:354-81.
31. Matthews BW. Comparison of the predicted and observed secondary structure of T4 phage lysozyme. Biochim Biophys Acta 1975;405: 442-51.

32. Baldi P, Brunak S, Chauvin Y, Andersen CA, Nielsen H. Assessing the accuracy of prediction algorithms for classification: an overview. Bioinformatics 2000;16:412-24.

33. Jensen A, Hohmann M, Künzel W. Dynamic changes in organ blood flow and oxygen consumption during acute asphyxia in fetal sheep. J Dev Physiol 1987;9:543-59.

34. Preziosi MP, Roig JC, Hargrove N, Burchfield DJ. Metabolic acidemia with hypoxia attenuates the hemodynamic responses to epinephrine during resuscitation in lambs. Crit Care Med 1993;21:1901-7.

35. Executive summary: neonatal encephalopathy and neurologic outcome, second edition. Report of the American College of Obstetricians and Gynecologists' Task Force on Neonatal Encephalopathy. Obs Gynecol 2014;123:896-901. 